\title{
La percepción sobre el ciberacoso del profesorado de secundaria de la Región de Murcia
}

\section{The perception of cyberbullying of Secondary School teachers of the Region of Murcia}

\author{
Víctor González Calatayud ${ }^{1}$, María Paz Prendes Espinosa ${ }^{2}$, José Antonio López Pina ${ }^{3}$ \\ ${ }^{1}$ Departamento Didáctica y Organización Escolar, Universidad de Murcia, España (victor.gonzalez@um.es) \\ ${ }^{2}$ Departamento Didáctica y Organización Escolar, Universidad de Murcia, España (pazprend@um.es) \\ ${ }^{3}$ Departamento Psicología Básica y Metodología, Universidad de Murcia, España (jlpina@um.es)
}

Recibido el 21 de octubre de 2016; revisado el 4 de noviembre de 2016; aceptado el 5 de noviembre de 2016; publicado el 2 de diciembre de 2016

\section{RESUMEN:}

El ciberacoso se ha convertido en uno de los principales problemas o riesgos en la red para los jóvenes. Los estudios hablan de hasta un $58 \%$ de estudiantes afectados por alguna forma de ciberacoso (Yang, Lin, y Chen, 2014). Sin embargo, pocos estudios se han centrado en conocer cómo perciben este problema los profesores, lo que llama la atención teniendo en cuenta que son los principales agentes, con los padres, para identificarlo e intervenir. Por ello, este estudio tiene como objetivo conocer la percepción del profesorado de secundaria en relación con el ciberacoso. Para ello se utilizó un cuestionario en inglés de una investigación previa y se utilizó el método de la doble traducción para su validación. Los resultados muestran que el profesorado subestima este problema. Además, admiten que no son capaces ni de identificar ni manejar los posibles casos de ciberacoso, aunque la mayoría llevaría a cabo alguna acción para detenerlo. Los profesores reclaman formación y una mejor orientación para el manejo de este problema, cuestión relevante que debe ser tenida en cuenta por las administraciones educativas y también por las universidades como responsables de la formación inicial de profesorado.

\section{PALABRAS ClAVE: CIBERACOSO, PROFESORADO, ENSEÑANZA SECUNDARIA.}

\section{ABSTRACT:}

Cyberbullying has become one of the main problems or online risks for young people. The studies report that up to $58 \%$ of students affected by some form of cyberbullying (Yang, Lin, y Chen, 2014). However, few studies have focused on understanding how teachers perceive this problem. Which is striking considering that are the main agents, with parents, to identify it and to intercede. Therefore, this study aims to know the opinion of secondary teachers. To achieve this objective a questionnaire -that already existed in English- is used, using the double translation method for validation. The results show that teachers underestimate the problem. In addition, they admit that they are unable to identify or manage possible cases of cyberbullying, although most do something to stop it. Teachers show a need for better training and guidance for handling this problem. This conclusion is relevant for administration and also for universities as responsible of initial teachers training. KEYWORDS: TEACHERS, CYBERBULLYING,
SECONDARY EDUCATION.

\section{INTRODUCCIÓN}

El hecho de que cada vez nuestra vida esté más ligada al uso de la tecnología lleva consigo que situaciones problemáticas que se dan en la vida real se lleven al mundo virtual. Un ejemplo de ello es el acoso escolar, en el que el uso de las TIC ha propiciado nuevas formas de acoso. Esta nueva forma de acoso se ha denominado ciberbullying o acoso cibernético, más comúnmente ciberacoso. En 
un sentido amplio el ciberbullying puede entenderse como "cualquier comportamiento realizado a través de medios electrónicos o digitales por individuos o grupos que mandan repetidamente mensajes hostiles o agresivos destinados a causar daño o molestia a los demás" (Tokunaga, 2010, p. 278).

Aunque el ciberacoso comparte algunas características con el acoso tradicional, como son el desequilibrio de poder y la intencionalidad, existen ciertas peculiaridades que diferencian ambos fenómenos: el anonimato (Moore, Nakano, Enomoto, y Suda, 2012), el efecto desinhibidor de Internet (Casale, Fiovaranti y Caplan, 2015), la amplitud de la audiencia (Sticca y Perren, 2013) y la repetición entendida como un solo mensaje o una sola foto puede ser compartido infinitas veces (Ybarra, Espelage y Mitchell, 2014). Estas diferencias han llevado a que cada autor intente conocer la prevalencia de esta problemática con un cuestionario propio que ha provocado que los porcentajes oscilen entre un $2.9 \%$ (Lam, Cheng, y Liu, 2013) y un $58.7 \%$ (Yang et al., 2014). En España, se ha detectado que alrededor del $30 \%$ de los estudiantes de secundaria ha sufrido algún tipo de ciberacoso (Estévez, Villardón, Calvete, Padilla, y Orue, 2010; Garaigordobil, 2015). Incluso en primaria el $30.6 \%$ de los alumnos ha observado alguna situación de ciberacoso (González y Calvo, 2012).

\subsection{Los profesores ante el ciberacoso}

La escuela es uno de los espacios más importantes de socialización de los menores y los docentes su principal agente educativo. Por ello, se les exige a los profesores ser los encargados de la prevención e intervención en conflictos y situaciones violentas entre los menores. Se les reclama, tanto por parte de las familias como de las instituciones educativas, saber identificar y actuar ante las situaciones de esta índole. Si le añadimos el hecho de que los menores pasan mucho tiempo en las redes, cobra gran relevancia mantener a los menores seguros cuando navegan por Internet (Compton, Campbell, y Mergler, 2014).

A pesar de que el ciberacoso puede ocurrir en otros lugares diferentes al centro escolar, se ha determinado que víctimas y acosadores se suelen conocer uno al otro en la escuela (Smith et al., 2008). Los estudios han confirmado que los compañeros de clase son un factor que puede predecir y cambiar las situaciones de ciberacoso (Festl y Quandt, 2013; Festl, Scharkow y Quandt, 2015). Por lo que es necesario que los profesores estén preparados y tengan la capacidad suficiente para tratar con esta problemática.

Mientras que la mayoría de los estudios se han centrado en examinar el ciberacoso desde la perspectiva del estudiante, solo unos cuantos estudios han tratado el tema de la percepción del profesorado ante el ciberacoso. Así, en algunos estudios los profesores, aunque fueron capaces de reconocer el problema y tomaron medidas, mostraron su inseguridad sobre si lo que hacían era lo correcto (Vandebosch, Poels y Deboutte, 2014). Además, se ha indicado que los profesores subestiman la prevalencia del ciberacoso, ya que para ellos es menos visible (Snakenborg, Van Acker y Gable, 2011) y porque los estudiantes no le hacen partícipes de esta problemática (Tokunaga, 2010). El hecho de que el profesorado no tenga mucho conocimiento sobre cómo tratar esta problemática ha sido considerado como una de las principales causas que llevan a los estudiantes a no contarles estas situaciones (Tenenbaum, Varjas, Meyers y Parris, 2011).

En general, los profesores afirman que el ciberacoso es un problema en su centro, siendo las profesoras las que más preocupación muestran (Eden, Heiman y Olenik-Shemesh, 2013). Li (2008) encontró que casi un tercio de los profesores pensaba que el ciberacoso era un problema en su escuela, aunque solamente un $13 \%$ indicaba sentirse capaz de identificarlo. Incluso en algún estudio se afirmó que el 59\% de los docentes mostró preocupación por este tema (Cassidy, Brown y Jackson, 2012). Por otra parte, los profesores de institutos percibían niveles más altos de ciberacoso que los profesores de los centros de primaria (Huang y Chou, 2013). En opinión de los alumnos, los profesores no saben manejar apropiadamente el tema del ciberacoso (Agatston, Kowalski, y Limber, 2007; DeSmet et al., 2014). Aparte de ser capaces de identificar la problemática, también se pide a los profesores que sean capaces de actuar ante estas situaciones. Sin embargo, Li (2008) encontró que solamente un $11 \%$ de los profesores opinaba que era capaz de manejar este problema.

De los estudios previos se puede llegar a la conclusión de que el nivel de formación del profesorado en relación a esta temática es escaso $(\mathrm{Li}$, 2008; Yilmaz, 2010), les faltan recursos para prevenir y tratar los casos de ciberacoso (ÁlvarezGarcía, Rodríguez, González-Castro, Núñez, y Álvarez, 2010). No solo son necesarias normas y regulaciones, sino que también se requiere de un conocimiento o competencias para tratar este problema (Altrichter y Posch, 2009). Así lo afirman los propios profesores, al indicar que se deberían 
realizar cursos de formación para el profesorado, diseñar planes para la prevención del ciberacoso y estrategias para ayudar a sus alumnos (DeSmet et al., 2015; Eden et al., 2013; Li, 2008). Y requieren de esos cursos porque, como indica Li (2008), los profesores no salen preparados de la universidad para saber manejar este problema. Igualmente, los profesores muestran la necesidad de más orientación profesional que les ayude a lidiar con esta problemática que no se ven en muchas ocasiones capaces de resolver (Vandebosch et al., 2014).

\section{METODOLOGÍA}

\subsection{Objetivo y diseño}

El objetivo principal de esta investigación es recoger la opinión del profesorado de secundaria ante las situaciones de ciberacoso que se dan entre los menores. Es decir, conocer cuál es la opinión del profesorado sobre la realidad de este problema. Concretamente, se pretende conocer la percepción sobre varios ámbitos: (1) saber si los profesores piensan que el ciberacoso es un problema en su centro; (2) conocer si los profesores son capaces de identificar y manejar las situaciones de ciberacoso; y (3) analizar si los profesores creen necesario realizar actividades para prevenir y actuar contra el ciberacoso.

Para conseguir este objetivo se realizó un estudio de diseño transversal descriptivo mediante una técnica de encuesta aplicada en el curso 2015/2016. Aunque en la investigación completa se ha utilizado también una encuesta para alumnos, en este artículo nos centraremos únicamente en los datos del profesorado.

\subsection{Participantes}

El estudio se ha realizado en la Comunidad Autónoma de la Región de Murcia con estudiantes y profesores de Enseñanza Secundaria Obligatoria. A partir de la selección de la muestra de alumnos con un procedimiento de muestreo estratificado por conglomerado, se ha seleccionado al profesorado por un muestreo por conveniencia, aplicando el cuestionario a los profesores de las aulas en las cuales se administró el cuestionario de alumnos. Este procedimiento nos ha permitido analizar posibles discrepancias entre ambas muestras. Se decidió escoger este nivel de Enseñanza Secundaria dado que existen estudios (Balakrishnan, 2015; Del Rey et al., 2016) que afirman que el mayor número de casos se producen en estas edades.
Al final se recogió un total de 83 cuestionarios de 8 centros repartidos por la Región de Murcia. De ese total un $41 \%(n=34)$ eran varones y el $45,8 \%(n=$ 38 ) eran tutores de alguna clase de ESO. La media de edad del profesorado de la muestra fue de 44.13 $(D T=7.79)$, con un intervalo que va desde los 27 hasta los 59 años.

\subsection{Instrumento}

Para conocer la percepción del profesorado sobre el ciberacoso se realizó un estudio previo de instrumentos de investigación sobre ciberacoso y se seleccionó el "Survey on school cyberbullying for preservice teachers" ( $\mathrm{Li}, 2008)$. Es un cuestionario con 15 ítems y una escala tipo Likert de 4 categorías que va de 1 "muy en desacuerdo" a 4 "muy de acuerdo". Además, se incluyó un bloque para recoger datos sociodemográficos del profesorado.

Al ser un cuestionario en inglés se utilizó el modelo propuesto por la International Test Commission para la traducción y adaptación (Muñiz, Elosua, y Hambleton, 2013). Para ello, en primer lugar, se pidió permiso al autor del cuestionario para su utilización, traducción y validación. Una vez obtenido se procedió a la realización de una doble traducción. Este procedimiento consiste en una primera traducción al español por un experto en inglés y una segunda traducción de esa versión en español a inglés nuevamente (retro-traducción). Después un comité se encargó de realizar una comparación de las dos versiones en inglés y se ajustó la versión en español para nuestro contexto intentando no perder el significado que se pretendía en la versión inicial. Con la muestra completa, el coeficiente de fiabilidad alfa de Cronbach obtenido para este cuestionario fue de .80 .

\subsection{Procedimiento}

Para la recogida de datos se solicitó, en primer lugar, permiso a la Consejería de Educación de la Región de Murcia, contando con la colaboración del Observatorio para la convivencia.

En una primera fase se utilizó un cuestionario en línea, anónimo y voluntario, usando la aplicación de la Universidad de Murcia destinada para ello (encuestas.um.es). Se le envió al equipo directivo de los centros participantes en la investigación un correo electrónico con el enlace para que a su vez se lo mandaran a todo el profesorado que impartía clase en Educación Secundaria Obligatoria.

Para completar la muestra, en una segunda fase se acudió personalmente a los centros y se administró el cuestionario en papel. 


\section{RESULTADOS}

Los profesores que participaron en el estudio afirmaron en gran proporción un desacuerdo $(n=67$, $80.7 \%$ ) con que el ciberacoso era un problema en su centro. En relación a este aspecto, no se encontraron diferencias entre varones $(M=2, D T=0.49) \mathrm{y}$ mujeres $(M=2.14, D T=.064), t(80.21)=-1.14 p=$ .257. Como se observa en la tabla 1 , el porcentaje varía de un centro a otro, aunque en uno de los centros, el porcentaje de acuerdo con que el ciberacoso es un problema en su centro fue muy elevado.

Igualmente, se halló un porcentaje alto ( $n=56$, $67.5 \%$ ) que indicó creer que sus alumnos no tienen problemas de ciberacoso. Sin embargo, casi tres cuartas partes $(n=61,71.5 \%)$ manifestó una preocupación por esta problemática.

Tabla 1. Opinión del profesorado sobre si el ciberacoso es un problema en su centro

\begin{tabular}{ccc}
\hline & $\begin{array}{c}\text { Muy en } \\
\text { desacuerdo o en } \\
\text { desacuerdo }\end{array}$ & $\begin{array}{c}\text { Muy de } \\
\text { acuerdo o de } \\
\text { acuerdo }\end{array}$ \\
\hline Centro 1 & $91.7 \%$ & $8.3 \%$ \\
Centro 2 & $83.3 \%$ & $16.7 \%$ \\
Centro 3 & $100 \%$ & 0 \\
Centro 4 & $38.5 \%$ & $61.5 \%$ \\
Centro 5 & $90.9 \%$ & $9.1 \%$ \\
Centro 6 & $60 \%$ & $40 \%$ \\
Centro 7 & $83.3 \%$ & $16.7 \%$ \\
Centro 8 & $95 \%$ & $5 \%$ \\
\hline Total & $80.7 \%$ & $19.3 \%$ \\
\hline
\end{tabular}

Asimismo, el porcentaje de aquellos profesores que indicaron creerse capaces de identificar los casos de ciberacoso $(n=34,40.9 \%)$ es inferior a los que no se ven capaces $(n=49,59.1 \%)$. Porcentajes similares se encontraron entre aquellos que afirmaron sentirse capaces de gestionar los casos de ciberacoso $(n=35,42.2 \%)$ y los que no $(\mathrm{n}=48$, $57.8 \%$ ). En cuanto a los centros, se observa como en algunos casos hay centros donde los profesores se ven capaces de identificarlo y manejarlo (tabla 2). En cuanto al sexo, no se encontraron diferencias en ninguna de las dos variables, aunque las mujeres muestran una media superior $(M=2.47, D T=0.71)$ en la variable de identificación que los hombres $(M$ $=2.21, D T=0.81)$, mientras que en cuanto a la gestión es prácticamente igual. Al mismo tiempo, prácticamente la totalidad de los participantes afirmó que haría algo para detener un caso de ciberacoso si conociese algún indicio de su existencia $(n=82$, $98.8 \%)$.
Tabla 2. Porcentaje de profesores que son capaces de identificar y gestionar el ciberacoso

\begin{tabular}{ccccc}
\hline & \multicolumn{2}{c}{ Identificar } & \multicolumn{2}{c}{ Gestionar } \\
& $\begin{array}{c}\text { En } \\
\text { desacuerdo }\end{array}$ & $\begin{array}{c}\text { De } \\
\text { acuerdo }\end{array}$ & $\begin{array}{c}\text { En } \\
\text { desacuer } \\
\text { do }\end{array}$ & $\begin{array}{c}\text { De } \\
\text { acuerdo }\end{array}$ \\
\hline Centro 1 & $50 \%$ & $50 \%$ & $66.7 \%$ & $33.3 \%$ \\
Centro 2 & $33.3 \%$ & $66.6 \%$ & $16.7 \%$ & $83.3 \%$ \\
Centro 3 & $50 \%$ & $50 \%$ & $75 \%$ & $25 \%$ \\
Centro 4 & $61.5 \%$ & $39.5 \%$ & $69.3 \%$ & $30.7 \%$ \\
Centro 5 & $45.5 \%$ & $55.5 \%$ & $36.4 \%$ & $63.6 \%$ \\
Centro 6 & $60 \%$ & $40 \%$ & $40 \%$ & $60 \%$ \\
Centro 7 & $66.7 \%$ & $33.3 \%$ & $66.6 \%$ & $33.4 \%$ \\
Centro 8 & $75.5 \%$ & $24.5 \%$ & $65 \%$ & $35 \%$ \\
\hline Total & $59.1 \%$ & $40.1 \%$ & $57.8 \%$ & $42.2 \%$ \\
\hline
\end{tabular}

Del mismo modo, la totalidad del profesorado cree que sus centros deberían desarrollar políticas que puedan evitar las situaciones de ciberacoso. Este porcentaje desciende al preguntarle si creen que se deberían usar horas de formación del profesorado para formarles en cuestiones relacionadas con el ciberacoso, siendo una gran mayoría la que opina estar de acuerdo ( $n=74,89.2 \%)$. También opinan que ellos mismos deberían organizar actividades en clase para trabajar este problema, ya que casi la totalidad afirmo estar de acuerdo $(n=75,90.4 \%)$.

Por otro lado, la mayoría de los encuestados $(n=$ 82, 98.8\%) opina que el equipo de orientación debería organizar tareas para trabajar el ciberacoso, mientras el porcentaje es algo menor de los que piensan que el equipo directo también debería organizar actividades para ello $(n=70,84.3 \%)$.

Otro de los aspectos que se les preguntó fue si creían necesario crear comités para investigar los casos de ciberacoso, encontrando que poco más de la $\operatorname{mitad}(n=48,57.8 \%)$ así lo cree. Igualmente, en su gran mayoría $(n=73,88 \%)$ consideran necesario hablar con las familias para tratar este problema del ciberacoso.

Por último, creen necesario que se conecte a los alumnos con los recursos a su disposición en la comunidad para los problemas que le puedan surgir en relación al ciberacoso $(n=74,89.2 \%)$, además de que en su totalidad opinan que es necesario asesorar a niños y adolescentes en asuntos relacionados con el ciberacoso.

\section{DISCUSIÓN}

Los resultados obtenidos muestran que en general el profesorado subestima la problemática del ciberacoso al indicar que el mismo no era un problema en su centro, al contrario que en estudios previos (Eden et al., 2013; Giménez-Gualdo, 2015; 
Li, 2008), lo que contrasta con la investigación previa sobre el ciberacoso en el que en muchos casos la prevalencia no es nada desdeñable (Estévez et al., 2010; Garaigordobil, 2015). Solamente en uno de los centros hay más profesores que opinan que el ciberacoso es un problema en su centro. El hecho de que el ciberacoso sea menos visible para el profesorado (Snakenborg et al., 2011) o que el alumnado se lo cuente menos (Tokunaga, 2010), pueden ser causas del porqué se subestime. A pesar de ello, la mayoría de los profesores mostraron preocupación por el tema. Al contrario de lo hallado por Eden et al. (2013) y Yilmaz (2010), los cuales determinaron que las mujeres mostraron más preocupación, en nuestros datos no se encontraron diferencias significativas.

Los padres y la administración exigen a los profesores que sean capaces de resolver esta problemática. Sin embargo, en general los profesores sienten que no son capaces de poder identificar este problema y mucho menos manejarlo, lo que concuerda con los estudios en los que el alumnado opinaba que los profesores no sabían manejar este problema (Agatston et al., 2007; DeSmet et al., 2014), aunque en algunos centros hay más profesores que se ven capaces de manejarlo. Para que fueran capaces de identificar, manejar e incluso prevenir el ciberacoso los profesores necesitarían de una formación de la que carecen, tal y como muestran nuestros datos y como así se ha determinado también en estudios previos (ÁlvarezGarcía et al., 2010; Yilmaz, 2010), una formación que les permitiera prevenir, identificar y actuar. Es más, en la opinión del profesorado es necesario realizar actividades tanto por parte del profesor como por parte del centro para trabajar este problema, además de involucrar a las familias.

\section{CONCLUSIONES}

Los resultados de esta investigación muestran que el profesorado no está preparado para una realidad que afecta cada vez más a los estudiantes. Ellos mismos son conscientes de que no sabrían identificarlo ni planificar una intervención adecuada. Por tanto, se hace necesario que la administración empiece a diseñar cursos de formación para enseñar a los profesores cómo actuar y también cómo prevenirlo. Y no solo la administración, sino que las universidades, como centros de formación inicial del profesorado, deberían dar formación al respecto. La formación se convierte así en una necesidad acuciante. A ello se añade el hecho de que, si los estudiantes no perciben que el profesorado sabe cómo manejar el problema, probablemente no lo manifiesten y ello aumentará la dificultad de su detección y de su resolución a través de la posible intervención del profesorado.

\section{LIMITACIONES}

Una de las principales limitaciones del estudio es la falta de una muestra más grande y aleatorizada que permita una generalización de los resultados. Además, el hecho de ser solamente un cuestionario cerrado impide conocer muchos aspectos interesantes como es conocer cómo han manejado situaciones de ciberacoso o saber qué problemas relacionados se han encontrado. Por tanto, para futuras investigaciones se pretende realizar un estudio más amplio, con una muestra representativa y aleatorizada y con preguntas abiertas que permitan conocer aspectos fundamentales para mejorar los planes de prevención y actuación de los centros. Además, pretendemos diseñar actuaciones de formación a partir de los datos obtenidos e informes de mejora para las instituciones implicadas en esta problemática.

\section{AGRADEMIMIENTOS}

Agradecemos al profesorado y al alumnado su participación en esta investigación, así como la colaboración de los equipos directivos. También agradecemos la autorización de la Consejería de Educación de la CARM, así como el apoyo del Observatorio de la Convivencia.

\section{REFERENCIAS}

Agatston, P. W., Kowalski, R. M. y Limber, S. P. (2007). Students' Perspectives on Cyber Bullying. Journal of Adolescent Health, 41(6), S59-S60. doi: 10.1016/j.jadohealth.2007.09.003

Altrichter, H. y Posch, P. (2009). Action Research, Professional Development and Systemic Reform. In S. Noffke y B. Somekh (Eds.), The SAGE Handbook of Educational Action Research (pp. 213-225). London, UK: SAGE Publications Ltd. doi: 10.4135/9780857021021.n20

Álvarez-García, D., Rodríguez, C., González-Castro, P., Núñez, J. C. y Álvarez, L. (2010). La formación de los futuros docentes frente a la violencia escolar. Revista de Psicodidáctica, 15(1), 35-56.

Balakrishnan, V. (2015). Cyberbullying among Young adults in Malaysia: the roles of gender, age and Internet frequency. Computer in Human behaviour, 46, 149-157. doi: 10.1016/j.chb.2015.01.021

Casale, S., Fiovaranti, G. y Caplan, S. (2015). Online Disinhibition. Journal of Media Psychology, 27(4), 170 177. doi: 10.1027/1864-1105/a000136

Cassidy, W., Brown, K. N. y Jackson, M. (2012). "Under the radar": Educators and cyberbullying in schools. School 
Psychology International, 33(5), 520-532. doi: 10.1177/0143034312445245

Compton, L., Campbell, M. A. y Mergler, A. (2014). Teacher, parent and student perceptions of the motives of cyberbullies. Social Psychology of Education, 17(3), 383-400. doi: 10.1007/s11218-014-9254-x

Del Rey, R., Lazuras, L., Casas, J.A., Barkoukis, V., OrtegaRuiz, R. y Tsorbatzoudis, H. (2016). Does empathy predict (cyber) bullying perpetration, and how do age, gender and nationality affect this relationship? Learning and Individual Differences, 45, 275-281. doi: 10.1016/j.lindif.2015.11.021

DeSmet, A., Aelterman, N., Bastiaensens, S., Van Cleemput, K., Poels, K., Vandebosch, H., ... De Bourdeaudhuij, I. (2015). Secondary school educators' perceptions and practices in handling cyberbullying among adolescents: A cluster analysis. Computers and Education, 88, 192201. doi: 10.1016/j.compedu.2015.05.006

DeSmet, A., Deforche, B., Hublet, A., Tanghe, A., Stremersch, E. y De Bourdeaudhuij, I. (2014). Traditional and cyberbullying victimization as correlates of psychosocial distress and barriers to a healthy lifestyle among severely obese adolescents--a matched case-control study on prevalence and results from a cross-sectional study. BMC Public Health, 14(1), 224. doi: 10.1186/1471-2458-14-224

Eden, S., Heiman, T. y Olenik-Shemesh, D. (2013). Teachers' perceptions, beliefs and concerns about cyberbullying. British Journal of Educational Technology, 44(6), 1036-1052. doi: 10.1111/j.1467-8535.2012.01363.x

Estévez, A., Villardón, L., Calvete, E., Padilla, P. y Orue, I. (2010). Adolescentes víctimas de cyberbullying: prevalencia y características. Psicothema, 18(1), 73-89.

Festl, R. y Quandt, T. (2013). Social Relations and Cyberbullying: The Influence of Individual and Structural Attributes on Victimization and Perpetration via the Internet. Human Communication Research, 39(1), 101-126. doi: 10.1111/j.1468-2958.2012.01442.x

Festl, R., Scharkow, M. y Quandt, T. (2015). The Individual or the Group: A Multilevel Analysis of Cyberbullying in School Classes. Human Communication Research, 41(4), 535-556. doi: 10.1111/hcre.12056

Garaigordobil, M. (2015). Cyberbullying in adolescents and youth in the Basque Country: prevalence of cybervictims, cyberaggressors, and cyberobservers. Journal of Youth Studies, 18(5), 569-582. doi: 10.1080/13676261.2014.992324

González, V. y Calvo, A. R. (2012). Ciberbullying en las aulas de primaria de Murcia. Comunicación presentada en las IV Jornadas Nacionales TIC y Educación (TICEMUR), Lorca, España. Resumen recuperado de http://ticemur.fintegra.org/comunicaciones/presenciales/06.pdf

Giménez-Gualdo, A. M. (2015). Cyberbullying. Análisis de su incidencia entre estudiantes y percepciones del profesorado (tesis doctoral). Murcia. Universidad de Murcia. Recuperado

de https://digitum.um.es/xmlui/handle/10201/46049

Huang, Y. y Chou, C. (2013). Revisiting cyberbullying: Perspectives from Taiwanese teachers. Computers and Education, 63, 227-239. doi: 10.1016/j.compedu.2012.11.023

Lam, L. T., Cheng, Z. y Liu, X. (2013). Violent online games exposure and cyberbullying/victimization among adolescents. Cyberpsychology, Behavior and Social Networking, 16(3), 159-165. doi: 10.1089/cyber.2012.0087
Li, Q. (2008). Cyberbullying in schools: An examination of preservice teachers' perception. Canadian Journal of Learning and Technology / La Revue Canadienne de L'apprentissage et de La Technologie, 34(2). Recuperado http://files.eric.ed.gov/fulltext/EJ1073833.pdf

Moore, M. J., Nakano, T., Enomoto, A. y Suda, T. (2012). Anonymity and roles associated with aggressive posts in an online forum. Computers in Human Behavior, 28(3), 861-867. doi: 10.1016/j.chb.2011.12.005

Muñiz, J., Elosua, P. y Hambleton, R. K. (2013). Directrices para la traducción y adaptación de los test: segunda edición. Psicothema, 25(2), 151-157. doi: 10.7334/psicothema2013.24

Smith, P. K., Mahdavi, J., Carvalho, M., Fisher, S., Russell, S. y Tippett, N. (2008). Cyberbullying: its nature and impact in secondary school pupils. Journal of Child Psychology and Psychiatry, 49(4), 376-385. doi: 10.1111/j.14697610.2007.01846.x

Snakenborg, J., Van Acker, R. y Gable, R. A. (2011). Cyberbullying: Prevention and Intervention to Protect Our Children and Youth. Preventing School Failure: Alternative Education for Children and Youth, 55(2), 88-95. doi: 10.1080/1045988X.2011.539454

Sticca, F. y Perren, S. (2013). Is cyberbullying worse than traditional bullying? Examining the differential roles of medium, publicity, and anonymity for the perceived severity of bullying. Journal of Youth and Adolescence, 42(5), 739-750. doi: 10.1007/s10964-012-9867-3

Tenenbaum, L. S., Varjas, K., Meyers, J. y Parris, L. (2011). Coping strategies and perceived effectiveness in fourth through eighth grade victims of bullying. School Psychology International, 32(3), 263-287. doi: 10.1177/0143034311402309

Tokunaga, R. (2010). Following you home from school: A critical review and synthesis of research on cyberbullying victimization. Computers in Human Behavior, 26(3), 277-287. doi: 10.1016/j.chb.2009.11.014

Vandebosch, H., Poels, K. y Deboutte, G. (2014). Schools and cyberbullying: problem perception, current actions and needs. International Journal of Cyber Society and Education, 7(1), 29-48. doi: 10.7903/ijcse.1149

Yang, S. C., Lin, C. y Chen, A. (2014). A Study of Taiwanese Teens' Traditional and Cyberbullying Behaviors. Journal of Educational Computing Research, 50(4), 525-552. doi: 10.2190/EC.50.4.e

Ybarra, M. L., Espelage, D. L. y Mitchell, K. J. (2014). Differentiating youth who are bullied from other victims of peer-aggression: the importance of differential power and repetition. The Journal of Adolescent Health: Official Publication of the Society for Adolescent Medicine, 55(2), 293-300. doi: 10.1016/j.jadohealth.2014.02.009

Yilmaz, H. (2010). An Examination of Preservice Teachers' Perceptions about Cyberbullying. Eurasia Journal of Mathematics Science y Technology Education, 6(4), 263-270. 